\title{
SISTEMA EDUCATIVO DE CHILE
}

\author{
Chilean Education System
}

\author{
Javier Ninahuaman, Henry Juan ${ }^{1}$ \\ Universidad Nacional Autónoma Altoandina de Tarma
}

\begin{abstract}
Resumen
El estudio es un análisis descriptivo cualitativo de diseño etnográfico a partir de los sucesos históricos y políticos del Sistema educativo de Chile, utiliza la ficha bibliográfica de instrumento y la técnica de observación y revisión documental. Se aborda las decisiones políticas y económicas de su gobierno que afectaron al estudiante, su familia y la sociedad en general. El Estado actúa como administrador de recursos alineándose a sus objetivos ideales, para ello propone programas, contenidos y formas de evaluar. El sistema se desarrolla con vouchers y copagos por criterio de eficiencia. Los estudiantes se manifestaron con protestas nacionales contra la municipalización y segregación social que trajo la clasificación entre escuelas municipales, colegios particulares subvencionados y establecimientos particulares pagados además del tipo de evaluación ejecutada por el SIMCE sumada a las desigualdades socioeconómicas, geográficas y de capacidad pese a las leyes de equidad. Aunque se da la garantía de acceso al aprendizaje escolar y sus resultados son verdaderos logros a nivel latinoamericano.
\end{abstract}

Palabras clave: Sistema educativo; Chile; América latina; aprendizaje; segregación escolar.

\begin{abstract}
The study is a qualitative descriptive analysis of ethnographic design from the historical and political events of the Chilean educational system, using the instrument's bibliographic card and the technique of observation and documentary review. It addresses the political and economic decisions of its government that affected the student, his or her family and society in general. The State acts as an administrator of resources aligning itself with its ideal objectives, for which it proposes programs, contents and forms of evaluation. The system is developed with vouchers and co-payments according to efficiency criteria. The students demonstrated with national protests against the municipalization and social segregation that brought about the classification between municipal schools, subsidized private schools and paid private establishments in addition to the type of evaluation executed by SIMCE added to the socioeconomic, geographic and capacity inequalities despite the laws of equity. Although there is a guarantee of access to school learning and its results are true achievements at the Latin American level.
\end{abstract}

Keywords: Education system; Chile; Latin America; learning; school segregation.

\footnotetext{
${ }^{1}$ Correspondencia: Henry Juan Javier Ninahuaman, hiavier@cip.org.pe, https://orcid.org/0000-0002-38644181
} 
Introducción

\section{Proceso en el tiempo}

El sistema educativo de Chile obedece a un proceso social en el tiempo, bajo el principio de la libertad de enseñanza desde 1874 se atiende como la posibilidad dada a los padres de elegir la educación de sus hijos y la libertad de crear y administrar colegios con financiación y apoyo del gobierno sin condiciones como a la educación pública.

El año 1973 se daba el golpe de estado contra Salvador Allende, se aprueba la Constitución de 1980 todo enfocado a la dictadura, pero basado en el mercado. De ello suscitó la municipalización de la educación básica, sus resultados fueron agudizar las diferencias de acuerdo a su capacidad económica, producto de ello el estado subvenciona por medio del bono educativo indistintamente si era colegio con o sin fines de lucro, iniciándose la segregación basado en criterios arbitrarios como las pruebas de conocimiento, economía, estatus social y hasta el credo religioso.

En 1990 se dan directrices neoliberales con la Ley Orgánica Constitucional de la Educación en un marco de concertación de partidos por la democracia, aunque con una fuerte influencia militar que conservaba intereses ideológicos conducentes a descentralizar y mantener la identidad nacional. Se perfila de esta manera el estado docente, creando una suerte de religión civil o cultura cívica, y se orienta a la privatización con apoyo militar. Como resultado se produjo el aumento de la cantidad de colegios privados y la calidad pues las subvenciones funcionan desde la base de la competencia entre escuelas públicas y privadas (Martínez \& Farías, 2018).

El sistema chileno se esquematiza en la educación privada sin subvención (de élite) siendo el 7\% de la población escolarizada, la educación privada subvencionada con financiación compartida entre el estado y los padres de familia en un $43 \%$, y las financiadas por el sector público concentrando un 50\%. El Estado hace el papel del que administra los recursos, como tal define los objetivos, programas, contenidos de enseñanza y la evaluación de los aprendizajes además de la normativa en función de su funcionamiento y financiación como las condiciones de infraestructura y estatutos (Martínez \& Farías, 2018).

En la década de 1990 se insertaron medidas de corrección al modelo consistentes en criterios de austeridad y eficiencia, parcializando de esta forma la provisión de los subsidios, haciendo que los beneficiarios aporten para su educación, este gasto es diferenciado entre los de ingresos altos pagan el costo real, los de ingreso medio pagan una parte y los pobres reciben gratuidad de servicio. A este sistema lo denominan voucher educativo que viene a ser el bono iniciado por Milton Friedman en 1955 con los argumentos del derecho de la elección del consumidor, el desarrollo personal como responsabilidad, competencia entre escuelas públicas y privadas y la igualdad de oportunidades por consecuencia de los tres principios anteriores.

El voucher se asigna por criterios de eficiencia y constancia y debe ser solicitado al Estado, los desembolsos al colegio son de acuerdo al número de estudiantes matriculados y su asistencia. El 2008 se reconocen las diferencias de origen y se implementa el subsidio diferenciado denominado Subsidio de Educación Preferencial - SEP - donde el Estado provee de mayores recursos a contextos de mayor vulnerabilidad socioeconómica, aunque luego del sétimo año de implementación los retos fueron mayores, por los criterios de inclusión - exclusión.

Desde el 2001 estudiantes de nivel secundario y medio se organizan y realizan manifestaciones políticas denominado el mochilazo inicializa su recorrido por la gratuidad del pasaje escolar, pasando luego a protestas contra el sistema chileno, ya al 2006 y 2011 se organizan en la Asamblea Coordinadora de Estudiantes secundarios - ACES - y la Coordinadora Nacional de Estudiantes secundarios - CONES- convocando a más de un millón estudiantes, sus siguientes demandas fueron sobre la gratuidad de la prueba de selección universitaria para los tres primeros quintiles de la población, la revisión de la Jornada escolar Completa - JEC - revisión de los débiles resultados académicos, estas demandas fueron etiquetadas como de corto plazo; empero fueron evolucionando a otras de mayor alcance demandando la desmunicipalización y centralización de los colegios públicos, la derogación de la Ley Orgánica Constitucional de Enseñanza - LOCE - y la educación gratuita, pública y de excelencia.

La nueva presidenta Michelle Bachelet (2006 - 2010) minimiza el conflicto estudiantil haciendo que este problema se agudice, aunque se negocia en el denominado Consejo Asesor para la Calidad de la Educación, logrando el 2007 la sustitución de la Ley General de Educación - LEG - por la Ley LOCE obligando a las corporaciones privadas con fines de lucro a dejar de serlas.

El 2011 a través de un ciclo de movilizaciones la Confederación de Estudiantes de Chile - CONFECHprotestan contra el costo excesivo de la educación superior por ser totalmente privada y el retraso del otorgamiento de las becas a estas se suman los estudiantes secundarios ACES y la CONES, estas protestas fueron bien articuladas utilizando de forma eficiente las redes informativas y la comunicación clásica y emergentes. 
Sebastian Piñera (2011-2015) promueve reformas educativas con la denominada Revolución Educativa destacando el Gran acuerdo Nacional de la Educación - GANE- y el Fondo por la Educación - FE- de 4000 millones de dólares, lo que causó mayores protestas y descontento pues no se enfocaban en las demandas reales del esquema mercantilista (Martínez \& Farías, 2018).

Chile cuenta con niveles de enseñanza básica o conocida como primaria basada en la propiedad pública o privada con financiamiento, divididos en escuelas municipales, colegios particulares subvencionados y establecimientos particulares pagados (Castillo Riquelme et al., 2018).:

\section{Agencia de Calidad de la Educación}

Con la ley 20529 se crea la Agencia de Calidad de la Educación de Chile con la visión de resguardar educación de calidad proveyendo evaluación, orientación e información confiable y útil para colaborar en el sistema de aseguramiento de la calidad, se rige con el valor de la calidad y la eficiencia, compromiso de colaboración, sentido de lo público y credibilidad, se despliega en macrozonas Norte: Arica y Parinacota, Tarapacá, Antofagasta, Atacama; Centro Norte: Coquimbo, Valparaíso, Metropolitana de Santiago, Libertador General Bernardo O’Higgins; Centro Sur: Maule, Ñuble, Biobío, La Araucanía; Sur: Los Ríos, Los Lagos; Austral: Aysén del General Carlos Ibáñez del Campo, Magallanes y de la Antártica Chilena.

Las Evaluaciones de desempeño hacen una clasificación de los establecimientos educativos en alto cuando sus estudiantes sobresalen respecto a los esperado, medio cuando el estudiante tiene resultados parecidos a los esperado, medio bajo cuando sus resultados son por debajo del nivel que se espera e insuficiente cuando el nivel es demasiado bajo, el cálculo tiene en consideración la distribución de estudiantes en los niveles de aprendizaje en $67 \%$, indicadores de desarrollo personal y social, resultados de pruebas del SIMCE y su progreso histórico de 2 o 3 mediciones 33\%, este índice es ajustado al contexto del estudiante.

Los estudiantes deben saber y poder demostrar los estándares de aprendizaje que considera el SIMCE siendo estos: insuficiente los que no logran demostrar consistentemente, elemental cuando se logra el currículo de manera parcial y adecuada cuando el nivel alcanzado es satisfactorio. (Agencia de Calidad de la Educación de Chile, 2020a; Aprueba ordenación de establecimientos educacionales reconocidos por el estado en conformidad al artículo 17 de la Ley N²20.529, nivel educación básica, 2018; Decreto 381, 2014; Ley 20529, 2011).

El gobierno pone a disposición herramientas de orientación al docente y estudiante en su página web donde se encuentran temas como conéctate, pregúntame, desafíate, jornadas de orientación territorial, talleres de orientación, infografías, fichas de orientación, herramientas de procesos de mejora (Agencia de Calidad de la Educación de Chile, 2020b). Además del Guía metodológica para el uso de datos donde se indican los pasos del guía, links de descarga del libro de la guía, descarga del cuaderno de trabajo de la guía, infografías, vídeos y lecturas recomendadas (Agencia de Calidad de la Educacioón de Chile, 2020).

El Sistema Nacional de evaluación de la calidad en educación - SIMCE -hace una prueba estandarizada a estudiantes de escuelas privadas, medio subvencionadas y totalmente pagantes, en las áreas de lenguaje, matemáticas, historia y ciencias naturales incluyéndose en los últimos años las áreas de inglés y educación física (Petour, 2015).

\section{Los tipos de Colegios}

La segmentación del mercado educativo en Chile fue dividida en tres grandes bloques:

Las escuelas municipales vienen a ser los lugares totalmente gratuitos y de naturaleza pública. Los estudiantes generalmente son calificados como vulnerables, tipificados por el Ministerio de educación chileno de acuerdo al grupo socioeconómico - GSE - en tipos bajo A y medio bajo B, constituyendo entre ellos el $84 \%$ de colegios municipales. Se estima que el $72 \%$ de colegios totales tipo A son municipales, donde los estudiantes tienen menos de 8 años de escolaridad, ingreso familiar bajo y vivencia de condiciones de pobreza, cuenta con un $79 \%$ vulnerables socialmente (Castillo Riquelme et al., 2018).

Las escuelas particulares subvencionados vienen a ser privados y cuentan con financiamiento estatal por control de vouchers designado por la asistencia de sus estudiantes. Sus estudiantes se caracterizan por ser diversos socioeconómicamente, reciben apoyo económico de las familias por medio del copago, en la actualidad ocupan el $55 \%$ de todo el nivel básico, de estos se estimó al 2013 que un 59\% calificaban como TIPO GSE medio - C - o GSE medio alto - D -, son moderadamente vulnerables socialmente, los padres o apoderados declaran tener enseñanza medio o estudios superiores (Castillo Riquelme et al., 2018).

Los colegios particulares no subvencionados o pagados ocupan alrededor del $8 \%$ de la matrícula dirigida principalmente a los sectores más pudientes, debido a su financiación exclusiva de los padres de familia, el $90 \%$ se 
tipifica como GSE -alto. Los apoderados cuentan con educación superior completa universitaria: Sus estudiantes vulnerables no superan los $11 \%$ (Castillo Riquelme et al., 2018).

\section{Método}

El estudio responde a un enfoque cualitativo, descriptivo de diseño etnográfico (Hernández Carrera, 2014; Martinez, 2004).

\section{Objetivos}

El objetivo del presente estudio es describir el Sistema educativo de Chile y su desarrollo histórico y político.

\section{Población y Muestra}

La población consta del sistema educativo de un país, de técnica no participante (Corbetta, 2007). La población muestra es el sistema educativo de Chile y fue elegida por conveniencia.

\section{Instrumento}

El instrumento utilizado es la ficha bibliográfica con la técnica de la observación y revisión documental o investigación de gabinete (Carrasco, 2006; Hernándes-Sampieri, Roberto Mendoza Torres, 2018; Niño Rojas, 2011).

\section{Procedimiento de recogida y análisis de datos}

Los datos fueron extraídos de artículos de la base de datos Scopus a partir de la plataforma Elseiver por el meta buscador Mendeley con opciones de búsqueda booleano or con la palabra clave Sistema, educación y Chile.

Los datos son sistematizados de acuerdo a los eventos históricos y políticos, haciendo la categorización de estructura.

El análisis de datos se hace por la técnica de registro, categorización y estructuración, teniendo en cuenta el análisis sintético por triangulación de datos de textos bibliográficos (Niño Rojas, 2011).

\section{Resultados}

En Chile se muestra segregación por diferencias de cultura, lengua y social, estos afectan a nivel emocional a los estudiantes y familia en general que se sumaron a protestas contra las políticas adoptadas desde 1984 respecto a la municipalización y segregación social por medio de pagos.

Chile obtiene resultados superiores respecto a los países latinoamericanos en las pruebas estandarizadas PISA y sostenidas por las pruebas SIMCE de calidad.

La política de Chile permitió la promulgación de diversas leyes para la inclusión social pues su misión es fortalecer la educación pública de calidad, aunque muestren problemas de conectividad virtual.

\section{Discusión y conclusiones}

A nivel mundial se generan políticas de garantía al acceso y promoción del aprendizaje escolar. De acuerdo a la categoría de Hobbes, la educación en Chile se encuentra en un campo no equitativo por ser demasiado amplio el número de factores implicados entre ellos los grupos de poder socioeconómico, la urbanidad, dominación y colonización.

Existen grupos minoritarios que son excluidos por diferencias culturales, lingüísticas, biológicas o sociales, bajo el lente de la teoría del reconocimiento para superación de barreras, participación y progreso desde el análisis del discurso de docentes. Las injusticias en este contexto dan realce al sujeto bajo la esfera de lo emocional, normativa y ética. Educación inclusiva sería por lo tanto una filosofía educativa, sobre la problemática de injusticia y desigualdad entre sujetos, reflexiona sobre la inclusión de sujetos o colectivos al debate educativo.

Chile en PISA 2018 obtuvo puntajes similares desde el 2015, en sus ítemes evaluados. En Lectura 2018, obtuvo 452 puntos, aunque menor al promedio de la OCDE y está sobre 31 países incluso de los Latinoamericanos 
(407) y por debajo de otros 41. En Matemática, obtuvo 417, menor al promedio de OCDE y mayor al de 18 países y menor que 53. Pero significativamente mayor que el promedio de Latinoamérica (387). En Ciencias Naturales, Chile llega a 444 puntos, igualmente menor al promedio de la OCDE pero superior a 30 de los sistemas y menor a otros 44. Chile se muestra significativamente mayor al promedio de los países latinoamericanos (403) (Agencia de Calidad de la Educación de Chile, 2020c; OCDE, 2017, 2019).

Estos resultados son loables a nivel latinoamericano pero a nivel social se hacen notar las brechas de desigualdades socioeconómicas, geográficas, de género, de capacidad y de edad principalmente en este sentido es necesario trabajar en la falta de trabajo colaborativo, la necesidad de formación, los recursos pedagógicos y el rol de los líderes educativos. Chile trabajó en leyes como:

Ley $\mathrm{N}^{\circ}$ 20.422/2010 sobre Igualdad de Oportunidades e Inclusión Social de personas con Discapacidad

Ley $N^{\circ} 20.609 / 2012$ que establece medidas contra la discriminación

Ley $\mathrm{N}^{\circ}$ 20.845/2015 que regula la admisión, elimina el financiamiento compartido y prohíbe el lucro en la educación.

Decreto n. 170/2009 que fija las normas para determinar los alumnos que presentan necesidades educativas especiales (NEE) y que serán eventuales beneficiarios de las subvenciones para educación especial

Decreto n. 83/2015 que aprueba criterios y orientaciones de adecuación curricular para estudiantes con NEE de educación parvularia y básica.

La Ley General de Educación n. 20.370 define la calidad educativa como una serie de objetivos y estándares que deben ser alcanzados por los y las estudiantes

Para el docente se muestra agrupación de los estudiantes en base a criterios evaluativos en estandarización de resultados y estrategia de aprendizaje.

Educar sería un reconocimiento normativo, la segregación es indeseada los docentes se centran en las característica del estudiante y no en los contextos pedagógicos. El ideal educativo es el sistema de recompensa y castigo, se mantiene la idea de que hay una sola forma de aprender (Alfaro \& Fernández, 2020).

El Ministerio de educación Chileno valora como eje del sistema escolar a la educación pública de calidad y la profesión docente moderna, para ello se intensifica la educación temprana, se integra nuevos servicios locales, incrementa la participación ciudadana, se otorga apoyo técnico, administrativo y financiero en campos de infraestructura, digitalización, talleres, deportivo y artístico, pedagógico, potencialidades docentes y directivas, participación y formación del estudiante. MINEDUC desde el 2015 pretende potenciar el área de convivencia y participación escolar además de asistir a los docentes de educación pública con programas de apoyo al maestro, a la gestión de los directores y su liderazgo con el fin de asegurar un mayor compromiso de profesores y directivos.

La ley general de educación del 12 de setiembre de 2009 indica el deber del Estado en garantizar educación de calidad y extendida a todo aquel habitante del país con el propósito de alcanzar el desarrollo espiritual, ético, moral, afectivo, intelectual, artístico y físico correspondiente la transmisión y cultivo de valores, conocimientos y destrezas.

El sistema educativo chileno tiene su base en los principios de calidad de la educación, equidad del sistema educativo, participación siendo informados y ser integrantes de la comunidad en su participación y principios de universalidad y educación permanente, autonomía, diversidad, responsabilidad, flexibilidad, transparencia, integración, sustentabilidad y la interculturalidad (Papic, 2018).

Los problemas principales de la educación en América Latina son la intensa actividad académica y el bajo impacto de estas, la escasa relevancia y calidad de la oferta educativa, la desvinculación de la realidad y el desconocimiento por parte de los docentes principiantes respecto a la realidad.

Al año 2006 los estudiantes de secundaria se organizaron políticamente para problematizar el modelo educativo abriendo paso a nuevas expectativas pues la consideraban como injusta y agraviante. Los puntos que observaban fueron el lucro en la innovación y la segregación social.

Chile promulga el 2016 la ley 20.911 de formación ciudadana a los establecimientos escolares e implica a la Universidad de Chile en la formación de docentes y directivos. El Salvador aprueba la ley General de educación que incluye el estudio moral, urbanidad y cívica como curso obligatorio esto fortalecerá capacidades de análisis y discusión para la toma de decisiones personal y colectiva. Ambos gobiernos suscribieron el proyecto de colaboración en la línea de acción del desarrollo de capacidades en formación ciudadana, convivencia, asistencia técnica, intercambio de experiencias y metodologías innovadoras, además de la transferencia de tecnologías y mecanismos de intercambio de conocimiento (Peña-Ruz, 2020).

Desde la época de la dictadura y postdictadura los logros educativos no fueron muy visibles, al contrario se profundizaron los procesos de segmentación socieducativa y desigualdad de oportunidades por el pago familiar para la educación, alejando los espacios de convivencia entre sectores. Aunque se revaloraron la participación y formación ciudadana como contenido transversal curricular, sin embargo los conocimientos cívicos y ciudadanía fueron tomados en poco. 
Los programas de estudios desde 1980 se pueden calificar como ideologización nacionalista por medio del conocimiento de las instituciones y el fortalecimiento de la identidad patriótica. A partir del 2012 y como consecuencia de las protestas estudiantiles el Estado se interesa por la problemática de la calidad, gratuidad y el fin al lucro por la educación.

El nivel de calidad educativa es recolectado a través de pruebas estandarizadas censales y lo realiza el sistema de medición de la calidad educativa - SIMCE- que amplía el alcance y medición a la convivencia escolar, hábitos de vida y participación ciudadana (Ramírez et al., 2015).

Los aspectos de calidad son medidos con cuestionarios estructurados en categorías de desempeño Alto, medio, medio bajo e insuficiente distribuidos por la Agencia de calidad de la Educación - ACE- entre sus dimensiones se encuentran los otros indicadores de calidad escolar - OIC- estos nuevos indicadores incluyen la participación y formación ciudadana midiendo el establecimiento hace promoción del sentido de pertenencia, participación, desarrollo de habilidades y actitudes para la vida en democracia.

Las familias restringen la participación política en la educación pues se consideran temas no pertinentes para la formación y ajenas a la participación de la institución educativa. La participación estudiantil no fue muy valorada y se limita a actividades de juego, cultura y deportivas (Castillo Riquelme et al., 2018).

La comunicación organizacional interna es de especial importancia para el desarrollo y mantenimiento de la organización en el marco de la educación de calidad, de mejora continua y educación de calidad, equidad y participación. Estamos en una era de postmodernidad caracterizada por la sociedad de consumo, bienestar y de la información, se configura de esta forma la aldea global donde la economía, el idioma y la tecnología es mundializada.

Sin embargo las herramientas automáticas especializadas como TAW y eXaminator, las páginas iniciales o home desarrolladas por el Ministerio de educación chileno abierta para el uso de personal educativo, docentes, padres de familia, estudiante so personas en general son calificados como poco accesibles y con problemas repetitivos como al falta de textos alternativos, imágenes, videos o elementos que no son claros interfiriendo así el proceso de información o apoyo a la educación (Ortiz Ruiz, 2019).

Pese a todos esos esfuerzos los docentes no se sienten valorados y no tienen confianza en el Estado, sin embargo si tienen la percepción que los estudiantes y comunidad los valoran. Para la toma de decisiones en educación los docentes son los últimos en ser consultados. Hasta los años 50 Chile fue el ejemplo en educación por la calidad de las instituciones y formación de docentes destacando la facultad de filosofía y educación de la Universidad de Chile, pero en la década del 70 las legislaciones deterioraron gradualmente esta característica al quitar la exclusividad a las universidades en formación de profesores, como la exigencia a los otros centros de estudio eran menores la valoración del profesor se relacionó a un rendimiento académico medio-bajo y bajo puntaje en la prueba estandarizada, a esto se sumó el bajo nivel de retribución económica, además está el abandono por su especialización y actualización (Hernández-Silva et al., 2017)

En el tema de inclusión se dictó la Ley 19284 el año 1994 de búsqueda plena de la persona con discapacidad y su pleno ejercicio de derechos constitutivos, además se dictó la norma del Ministerio de salud D.S. 2505 y 2542 sobre el reglamento de evaluación y calificación de la discapacidad, la ley 19284 del Ministerio de Educación en integración social, a esto suma el D.S. 40 y 47 del Ministerio de Urbanismo y Vivienda. El 2010 la Ley 20422 establece la igualdad de oportunidades e inclusión social de personas con discapacidad complementando la ley 19284.

Chile fundó la primera escuela especial de Latinoamérica el 1852 en Santiago dedicada a atender a niños sordos y mudos, luego en 1875 se implementa con la escuela para ciegos.

Desde 1946 se dictan normas dirigidos a personas con déficit mental, visual, trastornos de lectoescritura, cálculo y auditivos con la creación del Departamento de Educación especial o Diferencial del Ministerio de Educación, con el decreto 01-1998 y 374/1999 se garantiza la educación de los estudiantes hospitalizados, complementados por el decreto $89 / 1990$ sobre los planes y programas de estudio para discapacidad visual y el $86 / 1990$ para la discapacidad auditiva y el 815/1990 para autismo, disfasia severa o psicosis, el 87/1990 para la discapacidad intelectual, el DS 577/1990 para la discapacidad motora, el 637/1994 para educandos con déficit visual, el 291/1999 reglamenta el funcionamiento de los grupos diferenciales, el 1300/2002 para trastornos específicos del lenguaje, el 332/2011 determina edades mínimas para el ingreso a la educación especial, educación adulta y aceleración curricular, el 01398/2006para los procedimientos de licencia de enseñanza básica y de competencia a estudiantes con discapacidad, el 3600/1994 establece la creación de talleres para estudiantes mayores a 26 años con discapacidad y el 702/2000 orienta escuelas y aulas hospitalarias en aspectos técnico y administrativos. Con la 170/2010 se subvenciona a estudiantes con necesidades especiales (Estay et al., 2015).

La reforma educacional de Chile hace poca mención a la educación de las personas con discapacidad y necesidades educativas especiales pese a abordar cerca del $12 \%$ de la población latinoamericana y solo en Chile según el último censo asciende a 12.9\% siendo el 7.2\% leve, 3.2 moderada y 2.5 severa. 
La educación en Chile de una de las más costosas del mundo, costeado en su mayoría por los padres de familia por el sistema de copago y vouchers. A Chile se la califica como el país de los vouchers y que esto refleja la segregación nacional y lidera el ranking mundial de la organización para la cooperación y desarrollo económico en desigualdad con un coeficiente de Gini de $46.6 \%$.

Chile tiene un sistema educativo que segrega a las escuelas en pobres, clase media y los ricos, a raíz de las políticas económicas neoliberales de 1973 (Moreno-Doña \& Jiménez, 2014).

\section{Referencias}

Aprueba ordenación de establecimientos educacionales reconocidos por el estado en conformidad al artículo 17 de la Ley $N^{\circ} 20.529$, nivel educación básica, Pub. L. No. 3434, 186 (2018). http://docsta.agce.cl/docs/2018/actos_terceros/REX-3434.pdf

Agencia de Calidad de la Educación de Chile. (2020a). Agencia de Calidad de la Educación de Chile. https://www.agenciaeducacion.cl/evaluaciones/categoria-de-desempeno/

Agencia de Calidad de la Educación de Chile. (2020b). Herramientas de orientacion. Agencia de Calidad de La Educación de Chile. https://www.agenciaeducacion.cl/orientacion/herramientas-de-orientacion/

Agencia de Calidad de la Educación de Chile. (2020c). PISA 2018: Chile lidera resultados en América Latina en lectura, pero sigue bajo en promedio OCDE. Agencia de Calidad de La Educación de Chile. https://www.agenciaeducacion.cl/noticias/pisa-2018-chile-lidera-resultados-en-america-latina-enlectura-pero-sigue-bajo-el-promedio-ocde/

Agencia de Calidad de la Educacioón de Chile. (2020). Guía metodológica para el uso de datos. Agencia de Calidad de La Educacioón de Chile. https://www.agenciaeducacion.cl/orientacion/herramientas-deorientacion/guia-metodologica-uso-datos/

Alfaro, J. E., \& Fernández, V. H. (2020). El reconocimiento de las diferencias como fundamento para la educación inclusiva: la evaluación como barrera en el discurso docente. Revista Brasileira de Educação, 25. https://doi.org/10.1590/s1413-24782020250030

Decreto 381, Biblioteca del Congreso Nacional 17 (2014). http://archivos.agenciaeducacion.cl/categoria-dedesempeno/DTO-381_25-OCT-2013.pdf

Carrasco, S. (2006). Metodología de la investigación (San Marcos (ed.); Primera). San Marcos.

Castillo Riquelme, V., Rodríguez Garcés, C., \& Escalona Burgos, J. (2018). Participación, vida democrática y sentido de pertenencia según tipo de establecimiento educativo en Chile. Páginas de Educación, 11(2). https://doi.org/10.22235/pe.v11i2.1630

Estay, J. G., Vrsalovic Henríquez, V., \& Cabezas Cáceres, C. (2015). Personas con discapacidad y políticas públicas de inclusión educativa en Chile. Revista Facultad de Ciencias de La Salud UDES, 2(1). https://doi.org/10.20320/rfcsudes-201521-249

Hernándes-Sampieri, Roberto Mendoza Torres, C. (2018). Metodología de la investigación. Las rutas cuantitativa, cualitativa y mixta (McGraw-Hill Interamericana editores (ed.); 1st ed.). Mc GRaw Hill Education. https://www.ebooks7-24.com:443/?il=6443.,

Hernández-Silva, C., Pavez-Lizarraga, A., González-Donoso, A., \& Tecpan-Flores, S. (2017). ¿Se sienten valorados los profesores en Chile? Educación y Educadores, 20(3). https://doi.org/10.5294/edu.2017.20.3.6

Hernández Carrera, R. (2014). La investigación cualitativa a través de entrevistas: Su análisis mediante la teoría fundamentada. Cuestiones Pedagógicas: Revista de Ciencias de La Educación, 23, 187-210.

Martínez, E. G., \& Farías, R. Z. (2018). Formas de inclusión-exclusión en el sistema educativo chileno: el movimiento estudiantil secundario 2006-2011. Revista Brasileira de Educação, 23. https://doi.org/10.1590/s1413-24782018230077

Martinez, M. (2004). Ciencia y arte en la metodología cualitativa (Editorial Trillas (ed.); 1st ed.).

Ley 20529, Biblioteca del Congreso Nacional de Chile 1 (2011). www.leychile.cldocumentourl:https://www.leychile.cl/N?i=1028635\&f=2019-04-25\&p=LEYNúM.20.529

Moreno-Doña, A., \& Jiménez, R. G. (2014). Dictadura chilena y sistema escolar: "a otros dieron de verdad esa cosa llamada educación." Educar Em Revista, 51. https://doi.org/10.1590/s0104-40602014000100005

Niño Rojas, V. M. (2011). Metodología de la investigación: Diseño y ejecución (E. de la u (ed.); Primera).

OCDE. (2017). Marco de Evaluación y de Análisis de PISA para el desarrollo: Lectura, Matemáticas y Ciencias. OCDE Publishing, Versión pr, 97. https://www.oecd.org/pisa/aboutpisa/ebook - PISA-D Framework_PRELIMINARY version_SPANISH.pdf 


\section{Alborada de la Ciencia}

OCDE. (2019). Informe PISA 2018. In Catálogo general de publicaciones oficiales: publicacionesoficiales.boe.es/. https://www.observatoriodelainfancia.es/ficherosoia/documentos/5943_d_InformePISA2018Espana1.pdf

Ortiz Ruiz, Y. T. (2019). Accesibilidad en sitios web del Ministerio de Educación de Chile. Tendencias Pedagógicas, 33. https://doi.org/10.15366/tp2019.33.008

Papic, K. (2018). La Comunicación organizacional interna en la organización educativa. Foro Educacional, 30. https://doi.org/10.29344/07180772.30.1310

Peña-Ruz, M. (2020). Desarrollo profesional docente y educación a distancia. Una experiencia desde la cooperación Sur-Sur. Revista Saberes Educativos, 5. https://doi.org/10.5354/2452-5014.2020.57818

Petour, M. T. F. (2015). Validity and equity in educational measurement: The case of SIMCE. Psicoperspectivas, 14(3). https://doi.org/10.5027/PSICOPERSPECTIVAS-VOL14-ISSUE3-FULLTEXT-618

Ramírez, V. S. M., Nogués, F. P., \& Henríquez, S. S. (2015). ¿Cuál cable primero? El desarme del sistema educativo en Chile. Latinoamérica. Revista de Estudios Latinoamericanos, 60.

https://doi.org/10.1016/j.larev.2015.03.002 